\title{
Isolated temporalis muscle metastasis of renal cell carcinoma
}

\author{
Da Woon Lee ${ }^{1}$, \\ Hyeong Rae Ryu', \\ Jun Hyuk Kim ${ }^{1}$, \\ Hwan Jun Choi ${ }^{1}$, \\ Hyein $\mathrm{Ahn}^{2}$ \\ Departments of ${ }^{1}$ Plastic and \\ Reconstructive Surgery and ${ }^{2}$ Pathology, \\ Soonchunhyang University Cheonan \\ Hospital, Cheonan, Korea
}

\begin{abstract}
Isolated head and neck metastasis of renal cell carcinoma (RCC) is relatively rare and metastasis to the temple area is very rare. Here, we present the case of a 51-year-old man who was diagnosed with RCC 2 years earlier and had a contralateral metastatic temple area lesion. The patient who was diagnosed with renal cell cancer and underwent a nephrectomy 2 years ago was referred to the plastic surgery department for a temple mass on the contralateral side. In the operative field, the mass was located in the temporalis muscle with a red-to-purple protruding shape. Biopsy of the mass revealed a metastatic RCC lesion. Computed tomography imaging showed a lobulated, contoured enhancing lesion. Positron emission tomography/computed tomography imaging showed high-fluorodeoxyglucose uptake in the right temporalis muscle. The patient underwent wide excision of the metastatic RCC including the temporalis muscle at the plastic surgery department. Skeletal muscle metastasis of head and neck lesions is extremely rare in RCC. Isolated contralateral temporalis muscle metastasis in RCC has not been previously reported in the literature. If a patient has a history of malignant cancer, plastic surgeons should always consider metastatic lesions of head and neck tumors. Because of its high metastatic ability and poor prognosis, it is very important to keep this case in mind.
\end{abstract}

Keywords: Metastasis / Renal cell carcinoma / Temporal muscle

\section{INTRODUCTION}

Renal cell carcinoma (RCC) accounts for more than $3 \%$ of all malignant diseases [1]. RCC is known for its aggressive behavior and $25 \%$ to $30 \%$ of patients with RCC were found to have metastatic disease at the time of diagnosis and about a third of the RCC patients who receive a nephrectomy eventually develop metastatic disease with a median time to relapse of $15-18$ months [2]. Metastatic disease to the head and neck ranges

\section{Correspondence: Hwan Jun Choi}

Department of Plastic and Reconstructive Surgery, Soonchunhyang University Cheonan Hospital, 31 Suncheonhyang 6-gil, Dongnam-gu, Cheonan 31151, Korea E-mail: medi619@hanmail.net

This work was supported by the National Research Foundation of Korea (NRF) grant funded by the Korea government (MSIT) (2020R1A2C1100891), and was supported by Soonchunhyang University research fund.

Received February 10, 2021 / Revised February 16, 2021 / Accepted February 20, 2021 from $15 \%$ to $30 \%$. The most common sites of RCC metastases are the lungs (76\%), local lymph nodes (66\%), bone (42\%), liver (41\%), and head and neck (15\%), with approximately $1 \%$ transferring to the head and neck in the absence of other metastases [3]. In the case of RCC, there are many reported cases of cutaneous metastasis after nephrectomy, and cases of atypical metastatic lesions have been reported in recent decades [2]. However, the incidence of skeletal muscle metastases of RCC is reported to be less than $1 \%$ of the total hematogenous metastases of RCC [3]. The reported musculoskeletal metastatic lesions were localized to surrounding structures such as the psoas muscle [4]. Metastasis to the head and neck is extremely rare, and isolated temporalis muscle metastasis of RCC has not been previously reported in the literature. Most of the lesions with distant metastases are ipsilateral, contralateral craniofacial muscle metastasis of RCC has also not been reported previously. In this 
article, we present the case of a 51-year-old man diagnosed with temporalis muscle contralateral metastasis from RCC 2 years after surgery.

\section{CASE REPORT}

A 51-year-old man presented with a palpable mass at the right temple area. The patient's history revealed that 25 months earlier, a left renal mass was incidentally discovered on ultrasound through a routine medical examination. The mass was diagnosed as RCC. Therefore, the patient was admitted to the urology department of our hospital and underwent laparoscopic radical nephrectomy. After staging, the patient was diagnosed with stage I RCC (T1, N0, M0). During the routine follow-up

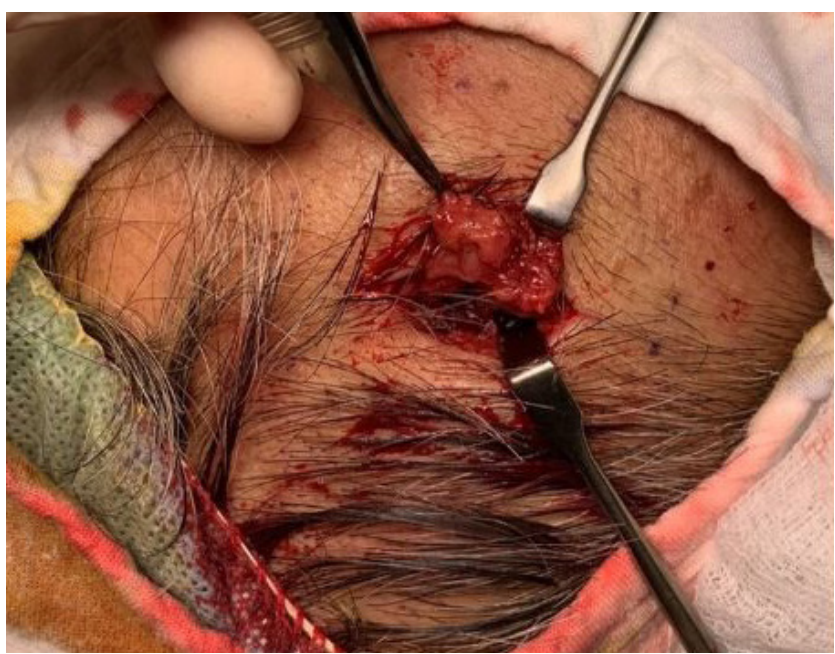

Fig. 1. Photograph of the intraoperative finding. An irregularly shaped mass with heterogeneous high vascularity was found in the temporalis muscle and attached to the periosteum. period after nephrectomy, no recurrence or metastasis was found on the bone scan or abdominal magnetic resonance imaging for several years. However, a palpable mass of the right temple area was observed three months before the latest urology department visit. The patient was referred to the plastic surgery department for a temporal mass biopsy.

Upon physical examination, there was a hard, red-to-purple, protruding mass on the right temple area. The patient had no specific history of trauma in the head and neck region and no other history of malignancy except for RCC. The patient underwent partial excision and biopsy. The intraoperative findings revealed an irregularly shaped mass with high vascularity in the temporalis muscle. Some necrotic tissue was also observed inside the mass. The mass was surrounded by the temporalis muscle and attached to the periosteum of the temporal bone (Fig. 1). The biopsy revealed metastasis of RCC. Our team conducted a radiological work-up for the metastatic temple lesion. Computed tomography (CT) imaging showed a $1.5-\mathrm{cm}-$ sized, lobulated, contrast-enhancing lesion with an irregular contour and high vascularity in the right temporalis muscle (Fig. 2A). Positron emission tomography/CT (PET/CT) imaging showed high-fluorodeoxyglucose (FDG) uptake in the right temporalis muscle, confirming metastatic cancer from RCC and right adrenal gland without recurrence at the left nephrectomy site (Fig. 2B and C). Ultrasonographic imaging showed a 2.4-cm-sized echogenic mass in the temporalis muscle with high vascularity in the peripheral portion. A month later, the patient underwent wide excision for the metastatic RCC including the randomized temporalis muscle and adjacent periosteum. The safety margin was roughly set to $7 \mathrm{~mm}$. In the first frozen biopsy sample, metastatic RCC cells were found in the temporalis muscle margin, and additional wide excision includ-
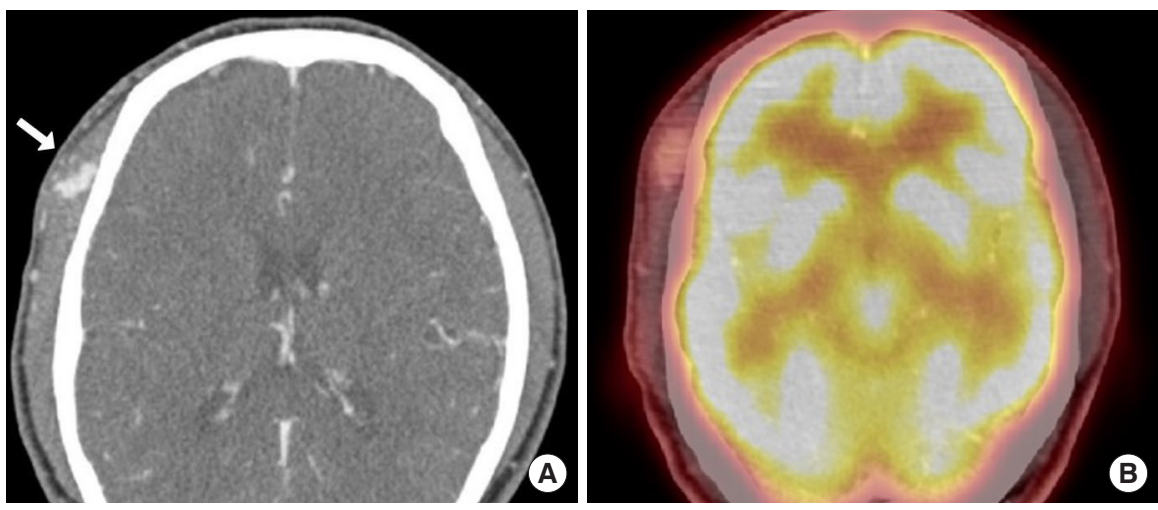

Fig. 2. (A) Computed tomography (CT) imaging showed a lobulated, contoured, enhancing lesion in the right temporalis muscle (white arrow). (B) Positron emission tomography (PET)/CT imaging shows an enhancing lesion with mild focal fluorodeoxyglucose (FDG) uptake in the right temporalis muscle. (C) PET/CT imaging shows high-FDG uptake in the temporalis muscle without recurrence at the left nephrectomy site (black arrow).

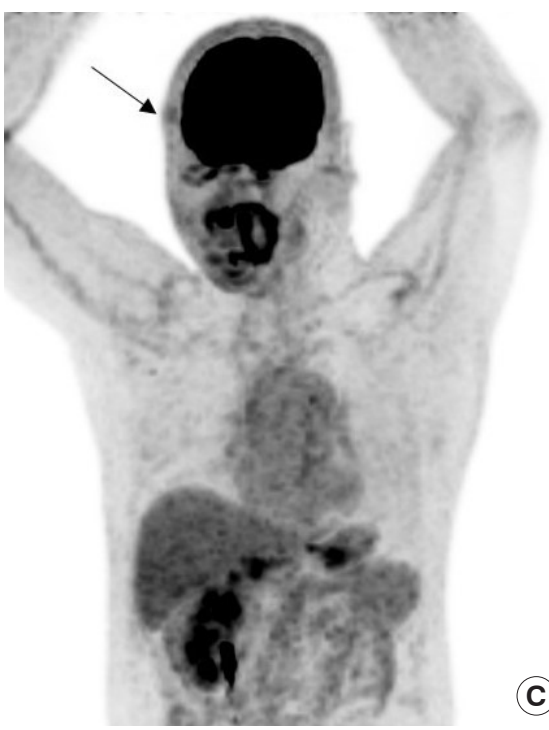



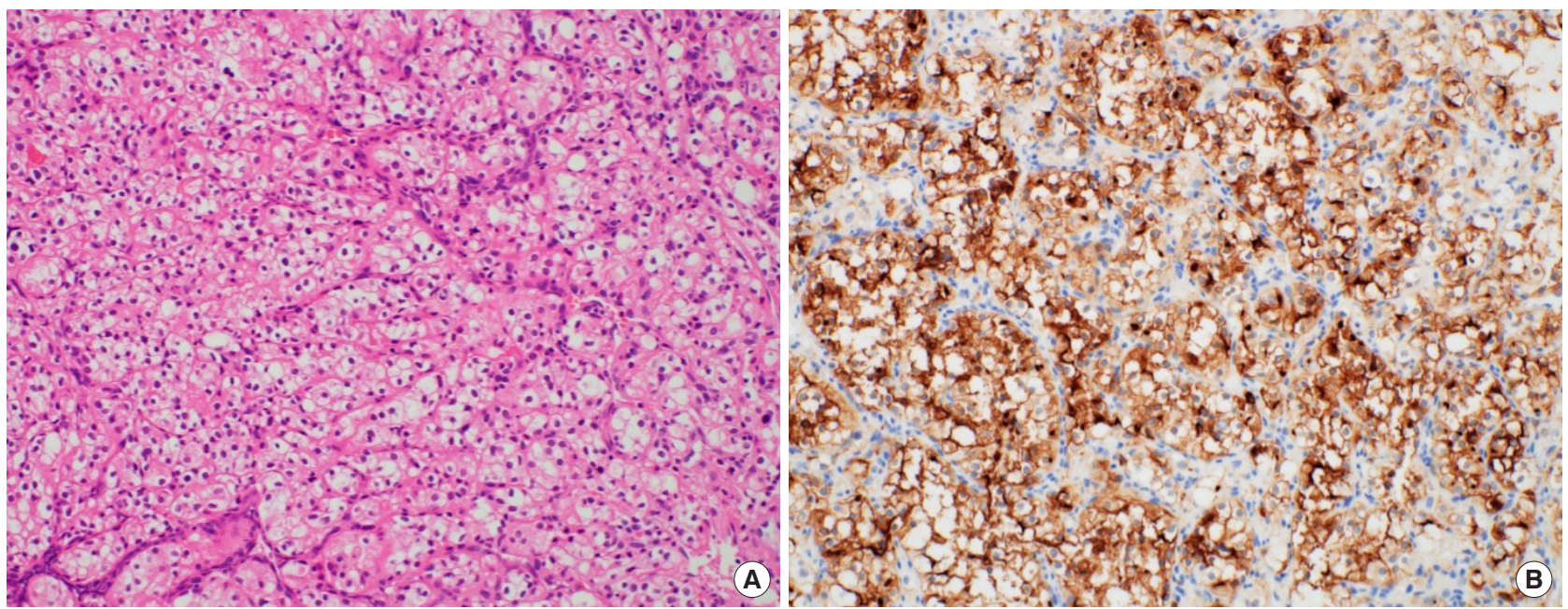

Fig. 3. Histopathologic findings. (A) The tumor cells have the same morphologic features as renal cell carcinoma (H\&E, $\times 200)$. (B) Immunohistochemical staining for CD10 revealed membranous and cytoplasmic expression in the tumor cells $(\times 200)$.

ing the temporal bone periosteum was performed. The second frozen biopsy revealed metastatic cancer cell-free margins in all directions of the removed mass. In the permanent pathology report, hematoxylin and eosin staining showed the infiltration of tumor cells into the adjacent soft tissue. The tumor cells had abundant clear cytoplasm and vesicular nuclei in compact architecture, showing the same morphologic features as RCC (Fig. 3A). Immunohistochemical CD10 staining revealed membranous and cytoplasmic expression in the tumor cells (Fig. 3B).

Finally, the patient was diagnosed with stage IVb clear cell RCC (T1, N0, M1). Since surgery, the patient has undergone molecular targeted therapy (pazopanib), with no evidence of systemic recurrence 4 months after surgery.

\section{DISCUSSION}

RCC usually occurs between the fifth and seventh decades of life and is twice as common in males [5]. Approximately, threefourths of all RCC detected is clear cell RCC. RCC is known to metastasize via hematogenous and lymphatic dissemination [5]. The first major route is through the venous system. Tumor cells in the renal vein may spread to the inferior vena cava, then eventually, into the lungs. So, pulmonary metastasis frequently occurs along this route. Tumor cells can also bypass pulmonary capillary filtration altogether via Batson plexus (cerebrospinal venous system), a network of valveless veins that connect the deep pelvic veins and thoracic veins to the vertebral column or brain without traversing the lungs $[6,7]$. This seemed to be the case in our patient, given the absence of lung involvement.

One of the most widely cited studies of RCC metastasis distri- bution is derived from the National Inpatient Sample [8]. In this study, 11,157 patients with metastatic RCC were identified from 1998 to 2007. The most common site of metastases was the lungs. Adrenal metastases were noted in $9 \%$ of the patients and brain metastases occurred in approximately $9 \%$ of the patients [8]. According to Yang and Kang [9], there was one case of cutaneous metastatic RCC to the scalp. Cutaneous metastasis can be described as a rapidly growing, nodular, round-to-ovalshaped mass, which can vary in color from normal skin color to a red-purple color. It may be confused with a hemangioma or pyogenic granuloma [10]. Metastasis to the calvarial bone, which is an extremely rare case, can be described as protruding from the skull. In some cases, it can compress the brain, causing signs of intracranial hypertension or even neurologic symptoms [11]. Craniofacial muscle metastasis is very rare in RCC. Isolated temporalis muscle metastasis for RCC has not been previously reported in the literature. Because metastatic cancer cells of the temporal muscle do not have lymphatic vessels, they are relatively more likely to metastasize remotely through hematogenic spreading [12]. It is also unique in that tumor cells usually spread ipsilaterally, whereas in this case tumor cells spread contralaterally. Patients are more likely to develop systemic metastases in the future, since the lesions have metastasized contralaterally.

Considering the round shape, the aspect, and radiologic finding of a mass, we initially suspected lipoma, hemangioma, or a sebaceous cyst rather than a metastatic mass. The possibility of metastatic cancer was thought to be very low at first. However, the biopsy revealed that it was clear cell-type metastatic RCC. This suggests that the authors had overlooked the possibility of malignancy of the tumor due to its benign looking clinical find- 
ings. When performing the excisional biopsy of a head and neck lesion, the plastic surgeon should consider the patient's cancer history and the possibility of malignancy. In addition, craniofacial muscle metastasis should always be considered in patients with RCC, even well after the primary treatment. Because of the high metastatic ability and poor prognosis of RCC, it is very important to keep this case in mind. Also, it is important to know that RCC can spread contralaterally.

The prognosis of patients with metastatic RCC is poor; the 5 -year survival rate ranges between $15 \%$ and $30 \%$ in the case of a single metastasis and between $0 \%$ and $7 \%$ in patients with multiple metastases [13]. It can be diagnosed through various radiologic examinations such as $\mathrm{CT}, \mathrm{PET} / \mathrm{CT}$, and histologic examinations.

Surgical excision, radiotherapy, chemotherapy, and hormonal therapy can be used to treat metastatic RCC $[14,15]$. Recently, molecular-targeted therapy has been increasingly used as the best RCC treatment since the beginning of this century [16]. Although the median progression-free survival of patients undergoing molecular targeted therapy is reportedly 6-11 months, compared to 5-6 months in the previously applied immunotherapy, molecular targeted therapy remains palliative [17]. This means that even with targeted therapy, the wide excision of metastatic lesions is also important. However, in most patients, targeted therapy is usually palliative treatment because of the late detection of metastatic lesions and poor prognosis. This is also due to the lack of communication between urologists who mainly deal with the abdomen, plastic surgeons who deal with facial lesions, and ear, nose, and throat (ENT) doctors. However, this patient was referred to a plastic surgeon within a few months of the appearance of the temporal mass, and the prognosis was improved by removing the metastatic tumor relatively early. Therefore, if the diagnosis of facial metastatic RCC is delayed, the cancer stage of RCC may be delayed at the same time. Thus, the delayed diagnosis of a patient with metastatic RCC can lead to a critical situation.

The timing of the first image scanning after treatment varies among the reported studies. While there are no formal recommendations, CT has been suggested at 1, 3, 6, and 12 months. After the first year, it has been further suggested that scans are performed every 6 or 12 months. It is not yet known for how long this should continue, but 5 years has been recommended [18].

In the case of RCC patients, a newly occurring mass anywhere in the body should be carefully evaluated. In the case of RCC, metastatic lesions may appear at any time after nephrectomy. These unusual metastases may be seen 2 years after radical nephrectomy. Although craniofacial muscle metastases indicate progression, disease-free follow-up is possible after appropriate surgical excision. Surgeons who operate on head and neck lesions should always operate with the potential of metastatic lesions in mind when performing an excisional biopsy in patients with a history of malignant cancer.

\section{NOTES}

\section{Conflict of interest}

No potential conflict of interest relevant to this article was reported.

\section{Ethical approval}

The study was approved by the Institutional Review Board of Soonchunhyang University Hospital (IRB No. 2020-12-051) and performed in accordance with the principles of the Declaration of Helsinki. Written informed consent was obtained.

\section{Patient consent}

The patient provided written informed consent for the publication and the use of his images.

\section{ORCID}

Da Woon Lee https://orcid.org/0000-0002-6969-5643 Hyeong Rae Ryu https://orcid.org/0000-0003-0962-2407 Jun Hyuk Kim https://orcid.org/0000-0002-0266-8480 Hwan Jun Choi https://orcid.org/0000-0002-0752-0389 Hyein Ahn https://orcid.org/0000-0001-9221-672X

\section{Author contribution}

Conceptualization: HJC. Resources: HA. Supervision: DWL, JHK. Writing - original draft: DWL. Writing - review \& editing: HRR. Approval of final manuscript: all authors.

\section{REFERENCES}

1. Singh J, Baheti V, Yadav SS, Mathur R. Occult renal cell carcinoma manifesting as nasal mass and epistaxis. Rev Urol 2014; 16:145-8.

2. Hussain F, Yedavalli N, Loeffler D, Kajdacsy-Balla A. Solitary parotid metastasis 8 years after a nephrectomy for renal cell carcinoma. J Community Hosp Intern Med Perspect 2016;6: 31950.

3. Zhang N, Zhou B, Huang Q, Chen X, Cui S, Huang Z, et al. Multiple metastases of clear-cell renal cell carcinoma to different region of the nasal cavity and paranasal sinus 3 times successively: a case report and literature review. Medicine (Baltimore) 2018;97:e0286. 
4. Wong BJ, Passy V, DiSaia P. Metastatic small cell carcinoma to the masseter muscle originating from the uterine cervix. Ear Nose Throat J 1995;74:118-21.

5. Bujons A, Pascual X, Martinez R, Rodriguez O, Palou J, Villavicencio $\mathrm{H}$. Cutaneous metastases in renal cell carcinoma. Urol Int 2008;80:111-2.

6. Brufau BP, Cerqueda CS, Villalba LB, Izquierdo RS, Gonzalez $\mathrm{BM}$, Molina CN. Metastatic renal cell carcinoma: radiologic findings and assessment of response to targeted antiangiogenic therapy by using multidetector CT. Radiographics 2013;33: 1691-716.

7. Jallu A, Latoo M, Pampori R. Rare case of renal cell carcinoma with mandibular swelling as primary presentation. Case Rep Urol 2013;2013:806192.

8. Bianchi M, Sun M, Jeldres C, Shariat SF, Trinh QD, Briganti A, et al. Distribution of metastatic sites in renal cell carcinoma: a population-based analysis. Ann Oncol 2012;23:973-80.

9. Yang HJ, Kang SY. Cutaneous metastatic renal cell carcinoma to the scalp Arch Craniofac Surg 2019;20:392-6.

10. Ferhatoglu MF, Senol K, Filiz AI. Skin metastasis of renal cell carcinoma: a case report. Cureus 2018;10:e3614.

11. Badri M, Gader G, Bahri K, Zammel I. Skull metastasis revealing a renal tumor: a case report and review of the literature. Int
J Surg Case Rep 2018;43:56-60.

12. Konishi M, Suzuki K, Iwai H. Hematogenous renal cell carcinoma metastasis in the postoperative temporal bone. Case Rep Oncol 2017;10:212-16.

13. Ralli M, Altissimi G, Turchetta R, Rigante M. Metastatic renal cell carcinoma presenting as a paranasal sinus mass: the importance of differential diagnosis. Case Rep Otolaryngol 2017; 2017:9242374.

14. Jayasooriya PR, Gunarathna IANS, Attygalla AM, Tilakaratne WM. Metastatic renal cell carcinoma presenting as a clear cell tumour in the head and neck region. Oral Oncol Extra 2004; 40:50-3.

15. Dimanovski J, Popovic A. Metastatic renal cell carcinoma. Acta Clin Croat 2000;39:171-3.

16. Escudier B, Gore M. Axitinib for the management of metastatic renal cell carcinoma. Drugs R D 2011;11:113-26.

17. Larkin J, Fishman M, Wood L, Negrier S, Olivier K, Pyle L, et al. Axitinib for the treatment of metastatic renal cell carcinoma: recommendations for therapy management to optimize outcomes. Am J Clin Oncol 2014;37:397-403.

18. Patel U, Sokhi H. Imaging in the follow-up of renal cell carcinoma. AJR Am J Roentgenol 2012;198:1266-76. 